\title{
Endogenous network formation in patent contests and its role as a barrier to entry*
}

\author{
Marco Marinucci ${ }^{\dagger} \quad$ Wouter Vergote V $^{\ddagger}$
}

October 23, 2009

\begin{abstract}
In a setting of R\&D co-opetition we study, by using an all-pay auction approach, how collaboration affects strategic decisions during a patent contest, and how the latter influences the possible collaboration network structures the firms can hope to form. The all pay auction approach allows us to 1) endogenize both network formation and R\&D intensities and 2) take heterogeneous and private valuations for patents into account.

We find that, different from previous literature, the complete network is not always the only pairwise stable network, even and especially if the benefits from cooperating are important. Interestingly, the other possible stable networks all have the realistic property that some firms decide not to participate in the contest. Thus, weak cooperation through network formation can serve as a barrier to entry on the market for innovation.

We further show that there need not be any network that survives a well known refinement of pairwise stability: strong stability, which imposes networks to be immune to coalitional deviations.
\end{abstract}

JEL Classification: L14, L24, O32

Keywords: Patent game, Networks, R\&D Cooperation, All-pay auction.

*The authors gratefully acknowlegde financial support from the Belgian Interuniversity Attraction Pole IAP/PAI P9/06. Wouter Vergote gratefully acknowlegdes financial support from the FNRS.

†Université catholique de Louvain, CORE, B-1348, Louvain La Neuve, Belgium and Facultés universitaires Saint-Louis, CEREC, 1000 Bruxelles, Belgium.

${ }^{\ddagger}$ Facultés universitaires Saint-Louis, CEREC, 1000 Bruxelles, Belgium and Université catholique de Louvain, CORE, B-1348 Louvain La Neuve, Belgium. 


\section{Introduction}

It is widely recognized that firms invest in $\mathrm{R} \& \mathrm{D}$ in order to increase their competitiveness and market power. Especially for high-tech sectors, firms are typically engaged in $\mathrm{R} \& \mathrm{D}$ contests in order to be the first, and maybe the only, to develop a new product/technology. In such an environment firms generally have the opportunity to develop some degree of collaboration. Most of the literature modelling R\&D competition has focused mainly on the two extremes of the collaboration spectrum: pure $R \& D$ competition (patent race, contest etc.) or full R\&D cooperation (R\&D Partnerships, Research Joint Ventures). ${ }^{1}$ Fewer attention has been devoted to intermediate forms of collaboration, in which firms cooperate to strengthen their joint position in R\&D contests, without sharing the benefits of winning the contest, however.

Nonetheless, the last decades witnessed an increasing number of R\&D partnerships even among competing firms (OECD [2001]). Second, it is shown (Greenlee [2005]) that firms adopt two types of cooperation: either firms build up Research Joint Ventures (RJV) where they coordinate resources (R\&D labs, scientists, etc.) and share the related results (technologies and products); or firms choose weaker forms of R\&D cooperation, like Cross Licensing, where partners just share their knowledge without the need of coordinating their $R \& D$ activity or sharing the $\mathrm{R} \& \mathrm{D}$ results. The existence of both types of cooperation is confirmed by Roijakkers \& Hagerdoon [2006] who show also that the share of weak partneships in the Biotech and Pharmaceutical sectors has been increasing during the last years.

Independently of the form of collaboration, it is clear that firms cannot keep up with their competitors without cooperating and competing with them at the same time. For example, Anand \& Khanna [2000] show that in the Electronics and Computer sectors competing firms usually cooperate via cross licensing even for technologies not yet developed.Cohen et al. [2002] find that not even high-tech but also low-tech Japanese firms usually cooperate through cross licensing and other forms of information sharing more often and more extensively than U.S. firms.

Given these stylized facts a first goal of this paper is to provide a theoretical framework to analyze sectors featured by "R\&D co-opetition", namely

\footnotetext{
${ }^{1}$ In particular, the latter stream of research has mainly focused on the welfare and/or the antitrust implication of Research Joint Ventures. See Caloghirou et al. [2003] \& Hagerdoon et al. [1999] for a survey on research partnerships.
} 
sectors where firms cooperate in $\mathrm{R} \& \mathrm{D}$ before competing with one another for the development of a new technology/product. In such a setting, two related questions arise quite naturally: First, how does (weak) cooperation benefit firms in the competition stage and how does that influence RGD activity? Second, given the impact cooperation has on RED activity, what are the likely patterns of cooperation that will emerge?

A great deal of literature has focused on (a part of) this issue, but, as far as we know, these two questions have not yet been analyzed jointly. Among the papers that analyze patent races and $R \& D$ cooperation at the same time (e.g. Martin [1995] and [2002], Stein [2008]) the contributions which are closer to ours are Goyal \& Joshi [2006] and Joshi [2008]. Both these papers propose a two stage game model where in the first stage firms cooperate in R\&D while in the second they participate in a patent race contest à la Loury [1979].

Goyal \& Joshi [2006] study endogenous network formation in a context of a patent race where the $R \& D$ investment is exogenously given. In other words question two has been answered fixing R\&D behaviour exogenously. Their main finding is that the complete network is the only one to be pairwise stable when the linking costs are low. On the other hand, Joshi [2008], in a similar setting, studies coalition formation game with endogenous R\&D effort. Joshi thus answers the two questions simultanously but coalition formation implies that firms strongly cooperate such that they both share the prize and coordinate the R\&D effort at the coalition level. ${ }^{2}$ Similarly to the previous paper, Joshi finds that, as the cost of participating in a coalition is low, the grand coalition is the only one to be pairwise stable. In both papers the value of the patent is treated to be the same for all firms and moreover it is common knowlegde. One contribution of our paper is to introduce heterogenous valuations for patents.

We answer the two above mentioned questions by developing a two stage game. In the first one, we consider a network game where firms can form links with one another where a link affects (increases) the expected value of the benefits of winning the patent game. In the second stage, firms are involved in a patent contest which is influenced by the network structure realized in the former stage. Differently from Goyal \& Joshi [2006] and Joshi [2008] we formalize such stage as an all-pay auction rather than a classical patent race.

The reason behind this is threefold. First, weak cooperation allows firms to increase their expected valuation for a patentable invention. This can be due to

\footnotetext{
${ }^{2}$ As we will see later on, Joshi motivates the choice of endogenizing the R\&D effort at the coalition level (rather than the individual one) because of a lack of tractability.
} 
the fact that firms assess the future market for their potential patented product differently, that they draw different marginal costs, etc. In order to capture this effect, we need to allow for the possibility that firms differ in their valuation for the benefits of obtaining a patent. When potential benefits are different among the various competitors, this will likely have an impact on the efforts dedicated to innovation. Moreover, since this valuation is usually private information, modeling the patent game as an all-pay auction with private information allows us to take this into account.

Second, since we wish to model the incentive to form weak R\&D links before entering into the patent game, the expected payoffs of the latter will play a major role in deciding which links to form. In order to perform this backward induction approach, the expected payoffs of the patent game need to be tractable. As has been pointed out by Joshi [2008] in a model of coalition formation in a race, when research intensities are chosen non-cooperatively in a classic patent race in which all firms equally value the benefits from the patent the equilibrium is no longer tractable. The all-pay auction with private information allows us to circumvent this problem by using the results of Parreiras and Rubinchik [2006].

Third, from a methodological point of view, as has been pointed out by Baye and Hoppe [2003] there is quasi-equivalence ${ }^{3}$ between classic patent races and rent seeking games, of which all pay auction is a special case through assuming that the firm with the highest $R \& D$ effort wins the patent game with probability one. $^{4}$

Hence, even though modelling the patent game as an all pay auction may seem, at first sight, an oversimplifaction, it allows us to take a more general approach by taking heterogenous and private valuations for a patent into account, while maintaining the spirit of the classic patent race (Loury) and, by doing so, study the stability of weak R\&D networks.

Once we have answered the above questions, it will be clear that when a firm decides to engage in $R \& D$ cooperation with a firm this will affect the expected profits of all its (potential) rivals in the $\mathrm{R} \& \mathrm{D}$ contest. Network formation makes a firm 'stronger' with respect to others and this may lead some rivals to decide not to invest in R\&D by exiting the market for innovation. Hence cooperation through networks has the potential to act as a barrier to entry. Given this we wish to know if this potential barrier to entry can arise in equilibrium. If the answer is yes, then we provide an alternative explanation for why some firms

\footnotetext{
${ }^{3}$ In fact, in the limit where the interest rate is equal to zero, there exists an isomorphism between the classic patent races and rent seeking contests.

${ }^{4}$ One could weaken the probability one assumption by mixing the all pay auction with a lottery, but this would only complicate the analysis without changing the qualitative results.
} 
may decide not to incur R\&D efforts, even though all firms started off on equal foot. In this situation, one may worry about a potential negative effect of weak cooperation: by creating barrier of entry on the market for innovation, does it reduce efficiency?

Our main results are as follows. First we show that our set up allows us to endogenize both R\&D effort and network formation. Second, like Goyal \& Joshi [2006] and Joshi [2008] we find that the complete network is always pairwise stable as the cost of forming a link is not too high. However, when the benefit of forming a link (i.e. a partnership) is large enough there exist asymmetric pairwise stable networks which have the dominant group architecture: a non empty set of firms does not participate to the contest while all participating firms form links with one another. Therefore, when cooperation is of high strategic importance, it becomes possible that some firms decide, in equilibrium, NOT to participate in the R\&D contest, even when the cost of linking is negligible. As mentioned above, the economic intuition behind our results is rather straightforward. Network formation between firms leads to better prospects from a future invention. By adding a link with another firm a firm becomes a 'stronger' participant in the R\&D contest. Firms that have few links can become so 'weak' that, for any possible valuation they may draw for the future invention (the patent), the marginal benefit from any positive $R \& D$ amount is always lower than the marginal cost, which makes them decide not to participate in the contest. Hence link formation between some firms can form a barrier to entry for other firms, strengthening the position of the former. In an example we show that a pairwise stable network with some firms opting out of the market for innovation can pareto dominate, from the point of view of the firms, the network formed by the grand coalition. Hence the barrier to entry is not necessarily welfare reducing.

When both types of networks are pairwise stable, we wish to refine the solution concept by studying which network structures are strongly stable by imposing immunity to coalitional deviations (Jackson and van den Nouweland [2005]). Unfortunately strong stability proves to be too strong a refinement as we show, again by means of an example, that there need not be any network surviving it.

The rest of the paper is organized as follows: in the next section we introduce the R\&D cooperation stage (Section 2) followed by the competitive one (Section 3). In Section 4, we solve the model while in Section 5 we provide an example of the model with five firms. In Section 6 we study the strong stability issue while Section 7 concludes. 


\section{R\&D Cooperation}

Modeling Networks We consider a finite set of ex-ante identical firms $N=$ $1, \ldots, n$ with $n>2$. Then, a network game is a game where every firm $i \in N$ announces its intended link $s_{i j} \in\{0,1\}$ with all firms $j \neq i$. If firm $i$ wants to make a link with $j$, then $s_{i j}=1$ and 0 otherwise.

Since firm $i$ has to decide whether to link or not with all its competitors, then a strategy for a firm $i$ is given by $s_{i}=\left\{s_{i j}\right\}_{j \neq i}$, which is a $n-1$ vector taken from the strategy set $S_{i}$.

A link among firms $i$ and $j$ will occur if and only if $s_{i j}=1=s_{j i}$, namely when both agree to form the partnership. In the following we describe the existence of a link among the players $i$ and $j$ through a binary variable $i j$ such that

$$
i j= \begin{cases}1 & \text { if } i \text { and } j \text { are linked } \\ 0 & \text { otherwise }\end{cases}
$$

A strategy profile $s=\left\{s_{1}, \ldots, s_{n}\right\}$ induces a network $g(s)$ which can arise from the set of all the possible networks $\Gamma$. Therefore, a network $g=\{(i j)\}$ is a $N \times N$ matrix describing all the pairwise links between the firms. Let $N_{i}(g)$ be the set of firms that have a link with firm $i$ given the network $g$. Then, $\eta_{i}(g)=\left|N_{i}(g)\right|$ is the number of firms linked with $i$. To simplify the notation, $g+i j$ means that the link $i j$ is added to the network $g$. Similarly, $g-i j$ corresponds to the network $g$ without the link $i j$.

We say that there exists a path between $i$ and $j$ if either $i j=1$ or if there exists a sequence of $l$ distinct players $\left\{k_{1}, k_{2}, \ldots k_{l}\right\}$ such that $i k_{1}=k_{1} k_{2}=\ldots=$ $k_{l-1} k_{l}=k_{l} j=1$. Network $g^{*}$ is said to be a component of network $g$ if for all $i$, $j, i \neq j$ belonging to $g^{*}$, there exists a path between $i$ and $j$ and for $i \in g^{*}$ and $j \in g$, if $i j=1$ then $j \in g^{*}$.

The complete network $g^{c}$ is characterized by $\eta_{i}\left(g^{c}\right)=n-1$ for all $i \in N$. The empty network $g^{0}$ is characterized by $\eta_{i}\left(g^{c}\right)=0$ for all $i \in N$.

A network $g$ is said to have a dominant group structure when the component $N^{D}(g)=\left\{i \in N, \eta_{i}(g)>0\right\} \subsetneq N$ is complete and all $j \notin N^{D}(g)$ have no links: $\eta_{j}(g)=0$

Main assumptions and stability concept Once a network is induced, we assume that each firm pays a negligible but positive cost $c>0$ per link formed. Given a strategy profile $s$, the payoff of firm $i$ is given by

$$
\Pi_{i}\left(s_{i}, s_{-i}\right)=\pi_{i}(g(s))-c \times \eta_{i}(g(s))
$$


Given this framework strategy profile $s^{*}=\left\{s_{1} \ldots s_{n}\right\}$ is a Nash Equilibrium if

$$
\Pi_{i}\left(s_{i}^{*}, s_{-i}^{*}\right) \geq \Pi_{i}\left(s_{i}, s_{-i}^{*}\right) \quad \forall s_{i} \in S_{i} ; \quad \forall i \in N
$$

However, Nash Equilibrium in network theory is too weak a concept for modeling network formation insofar as it allows the existence of too many equilibrium networks. ${ }^{5}$ Therefore, we adopt a stronger stability concept proposed by Jackson and Wolinsky [1996]; pairwise stability

Definition 1 (Pairwise Stability) A network $g$ is pairwise stable (PWS) if the following two conditions hold

1. there exists a Nash Equilibrium that supports $g$

2. $\forall i j \notin g$ if $\pi_{i}(g+i j)>\pi_{i}(g) \Rightarrow \pi_{j}(g+i j)<\pi_{j}(g)$

Intuitively, the two conditions state that, starting from a network $g$, no firm wants to sever a link (condition 1) and no couple of firms want to form a new one (condition 2).

When there is more than one pairwise stable network structure, it is useful to consider a refinement introduced by Jackson and van den Nouweland [2005]; strong stability, which corresponds to the idea that a network should be immune to coalitional deviations.

Definition 2 (Strong Stability) A network $g$ is strongly stable (SS) if the following two conditions hold

1. there exists a Nash Equilibrium that supports $g$

2. There does not exist a set of players $N^{\prime} \subset N$ and a network $g^{N^{\prime}}(g)$ such that, $N^{\prime}$ can implement $g^{N^{\prime}}(g)$ from network $g$ such that $\forall i \in N^{\prime}$ : $\pi_{i}\left(g^{N^{\prime}}(g)\right) \geqslant \pi_{i}(g)$ with at least one $i \in N^{\prime}: \pi_{i}\left(g^{N^{\prime}}(g)\right)>\pi_{i}(g)$.

It is immediate that if a network is strongly stable, it is also pairwise stable. The reverse is not always true.

\section{$3 \quad$ R\&D Competition}

We now model how the formation of weak cooperation influences the (expected) payoffs during the patent contests.

\footnotetext{
${ }^{5}$ For example, the empty network is always a Nash Equilibrium: if no one has an incentive to make a link with $i$, then the best strategy for $i$ is to reject the link formation $\left(s_{i j}=0\right)$.

${ }^{6}$ Given network $g$,a set of players $N^{\prime} \subset N$, can implement a network $g^{\prime}$ from $g$ through deletion or adding of links solely by members of 'coalition' $N^{\prime}$.
} 
We assume that the creation of a new link $i j$ allows firms $i$ and $j$ to improve their expected prospects from a patentable invention. More precisely we model this by assuming that firm's $i$ valuation of the patent given a network $g, v_{i}(g)$, be uniformly distributed according to

$$
v_{i}(g) \sim \Delta_{i}(g)=U\left[0, \alpha_{i}(g)\right]
$$

where $\alpha_{i}(g)$ is the ex-ante maximal valuation of the patent given the network $g$. This means that the support of the distribution of values depends on the network $g$.

Given these assumptions each firm chooses an R\&D effort level $\sigma_{i} \in[0, \infty)$ in order to maximize her expected payoff from the all pay auction. We are looking for an equilibrium of effort levels which is increasing in the valuation of the firm. The expected payoff form firm $i$ is:

$$
\pi_{i}\left(\sigma, \phi_{i}, g\right)=\phi_{i} \prod_{j \neq i} \Delta_{j}\left(\phi_{j}(\sigma)\right)-\sigma
$$

Where $\phi_{j}(\sigma)$ is the valuation of contestant $j$ such that she also makes an effort equal to $\sigma$ : the inverse bidding function. The first order condition can be written as:

$$
\phi_{i} \sum_{j \neq i} \prod_{k \neq j, i} \phi_{k}(\sigma) \frac{1}{\alpha_{j}(g)} \phi_{j}^{\prime}(\sigma)=1
$$

The left hand side is the marginal benefit of an extra $R \& D$ effort: it is the valuation for the invention times the increase in the probability of winning the contest. The right hand side is the marginal cost. It could well be that a contestant's first order condition is negative, even for its highest possible valuation $^{7}$. In this case this firm will not enter the race. This intuition was confirmed formally by Perreiras and Rubinchik (2006) (condition 9 on page 18) as they derived the following participation condition on the distributions of the firms' valuations: contestant $i$ will not participate; $\sigma_{i}(v)=0 \forall v \in\left[0, \alpha_{i}(g)\right]$, if the following condition is satisfied:

$$
\alpha_{i} \sum_{j \neq i} \alpha_{j}^{-1}<(N-2)
$$

\footnotetext{
${ }^{7}$ Assume contestant $i$ has no links $\left(\phi_{i} \leq 1\right)$ then if all other contestants have links such that $\alpha_{j}(g)$ becomes very large for all $j \neq i$, then likely $\phi_{k}(\sigma) \phi_{j}^{\prime}(\sigma)$ will become very small compared to $\alpha_{j}(g)$. That is, in order to bid the same amount of contestant $i$ they need to draw a very low value $\phi_{j}(\sigma)$, compared to $\alpha_{j}(g)$, and hence by contemplating to increase any positive bid, contestant $i$ can only marginally change the probability of winning.
} 
Once again, the intuition behind this result is that for these firms that if they participate to the contest (exert a positive R\&D effort), the marginal benefit of increasing their effort (the increased probability of winning times their valuation) is always smaller than the marginal cost and hence hence they are better off not participating at all. In fact one can check that a firm $i$ will not participate, given the others are participating if its maximum valuation $\alpha_{i}(g)$ is lower than $(N-1) / N$ times the harmonic mean of the maximum valuations $\alpha_{j}(g)$ of all firms $j$ who participate. This result mirrors the result obtained in Hillman and Riley (1989) for a linear Tullock contest without private information. The driving force behind the potential barrier to entry are the asymmetric valuations that stem from a certain network structure. The more links one has, the more likely some firms will drop out, leaving fewer competitors for the prize.

However, this does not necessarily mean that the network $g$ in which a firm $j$ exits the race will be pairwaise stable. It could well be that there is always another firm $i$ such that the network $g+i j$ is mutually beneficial for $i$ and $j$.

In order to answer this question we need to take a look at the payoffs of any given network. Given any network $g$ one can use condition 6 to eliminate all non-active participants and study the payoffs of the contest with the remaining firms. Suppose that there are $f(g) \leq N$ remaining participants, than the first order conditions can be manipulated (see Parreiras and Rubenchik (2006) for details) in order to obtain:

$$
\sigma_{i}(v)=\frac{f(g)-1}{\sum_{j} \alpha_{j}^{-1}}\left[\frac{v}{\alpha_{i}}\right]^{\frac{1+\kappa}{\kappa_{i}}}
$$

Where $\kappa_{i}=\frac{\sum_{j \neq i} \alpha_{j}^{-1}-(f(g)-2) \alpha_{i}^{-1}}{\sum_{j \neq i} \alpha_{j}^{-1}-(f(g)-2) \alpha_{1}^{-1}}$ and $\kappa=\sum_{i \neq 1} \kappa_{i}$

This yields expected payoffs :

$$
\pi_{i}=\left[\alpha_{i}-\frac{f(g)-1}{\sum_{j} \alpha_{j}^{-1}}\right] \frac{\sum_{j} \alpha_{j}^{-1}-(f(g)-1) \alpha_{i}^{-1}}{2 \sum_{j} \alpha_{j}^{-1}-(f(g)-1) \alpha_{1}^{-1}}
$$

Given these building blocks, we can summarize the timing of the patent contest in three steps:

STEP 1. The network $g$ determines the distributions $\Delta_{i}(g)$ of all firms.

STEP 2. Each firm decides whether to enter the contest or not. Following Parreiras \& Rubinchik [2006], a firm $i$ will participate to the race if and only if the following condition

$$
\Pi_{i}(N)>0 \Leftrightarrow \alpha_{i} \sum_{j \neq i} \alpha_{j}^{-1}>(N-2)
$$


is satisfied. Let $F(g): \Gamma \rightarrow N$ be a function that describes the set of firms who participate to the contest given network $g$. Define $f(g)=\# F(g)$

STEP 3. Each firm $i \in F(g)$ draws its private valuation $v_{i}$ and makes a non recoverable bid (proxy for R\&D effort). The highest bidder wins the contest.

\section{Solution}

Before we study if there are pairwise stable networks, we will make two simplifying assumptions. First, the support only depends on the amount of links a firm has: $\alpha_{i}(g)=\alpha_{i}\left(\eta_{i}(g)\right)$. Second, we assume that there exists a multiplicative effect $^{8}$ on the expected maximal value $\alpha_{i}$ in such way that:

$$
\alpha_{i}(g)=\alpha_{i}\left(\eta_{i}(g)\right)=\phi^{\eta_{i}(g)} \quad \phi>1
$$

In order to find out which partnership structures are pairwise stable, we solve the model by backward induction. Given the expected payoff functions obtained above we obtain for any network $g$ :

$$
\begin{aligned}
& \forall i \in F(g): \pi_{i}(g)=\frac{\alpha_{i}\left(\alpha_{i} A_{-i}-f(g)+2\right)^{2}}{\left(2 \alpha_{i} A_{-i}-f(g)+3\right)\left(1+\alpha_{i} A_{-i}\right)} \\
& \forall i \notin f(g): \pi_{i}(g)=0
\end{aligned}
$$

where $A_{-i}=\sum_{j \neq i}^{F(g)} \alpha_{j}^{-1}$.

Given this equilibrium behavior and payoffs in stage two, which network constellations are pairwise stable? In order to answer this question it is useful to consider the payoff of (a participating) firm $i$ when forming a link with firm $j$, given network $g$. Following Equation (10), the payoff of firm $i$ in the network $g+i j$ is

$$
\pi_{i}(g+i j)=\frac{\phi \alpha_{i}\left(\phi \alpha_{i}\left(A_{p}+\frac{1}{\phi \alpha_{j}}\right)-f(g+i j)+2\right)^{2}}{\left(2 \phi \alpha_{i}\left(A_{p}+\frac{1}{\phi \alpha_{j}}\right)-f(g+i j)+3\right)\left(1+\phi \alpha_{i}\left(A_{p}+\frac{1}{\phi \alpha_{j}}\right)\right)}
$$

Where $A_{p}=\sum_{p}^{F(g+i j) \backslash\{i, j\}} \alpha_{p}^{-1}{ }^{9}$

Looking at the linking strategy, it is straightforward to see that, given a network

\footnotetext{
${ }^{8}$ We thus assume the existence of a strong strategic incentive to cooperate which corroborates empirical observations mentioned in the introduction. Even if this strong cooperative environment exists, we will show complete network will not always be formed. We make these assumption in order to be able to compare expected payoffs between network $g$ and network $g+i j$ so as to be able to say something meaningful regarding pairwise stability. No doubt, our result could hold for more general relationships between a network and the distributions of valuations, but unfortunately, this comes at the cost of making general conclusions regarding pairwise stability.

${ }^{9} \mathrm{~A}$ non participating firm will prefer to abandon the race whenever the expected payoff from entering the race is non positive.
} 
$g$ and a negligible ${ }^{10}$ linking cost $c$, two firms will have an incentive to form a link if and only if both expected payoffs are strictly increasing

$$
\pi_{i}(g+i j)>\pi_{i}(g) \quad \text { and } \quad \pi_{j}(g+i j)>\pi_{j}(g)
$$

which implies also that both firms are willing to participate in the contest $g+i j$.

In particular, the decision to form a link is determined by the strenght of three effects. First, firms $i$ and $j$ benefit directly from improving their expected valuation of the patent. Second, since the new partnership strengthens the participation constraint of their competitors (Equation 9), partners benefit indirectly if other firms eventually decide to quit the race. Nevertheless, the formation of a link also generates a competitive effect as the linking partners become fiercer competitors, and as such a new link may be harmful for the partners. However, comparing equations (11) and (13) we find the following

Lemma 1 (Cooperative effect dominance) If firms $i$ and $j$ participate in $g$ and are not linked in $g$, then $i$ and $j$ always have an incentive to form the network $g+i j, \forall \phi>1$

$$
\pi_{i}(g+i j)>\pi_{i}(g) \quad ; \quad \pi_{j}(g+i j)>\pi_{j}(g) \quad \forall i, j \in F(g)
$$

Proof: See Appendix A.

In other words, the cooperative effect means that two non-connected competing firms always have an incentive to cooperate. It is also immediately seen that:

Corollary 1 The expected payoff of the patent game is increasing in the number of links.

Corollary 2 No firms that participate to the contest in the event of being linked, have an incentive to cut their link.

Consequently, we can conclude that

Proposition 1 The complete network $g^{c}$ is always pairwise stable.

Proposition 2 confirms the results found in Goyal \& Joshi (2006) and Joshi (2008): if the complete network (grand coalition) is formed, then no firms have an incentive to deviate. However, this does not exclude the existence of other pairwise stable networks. In order to confirm the uniqueness result we need

\footnotetext{
${ }^{10}$ In fact there is a threshold value of $c, \widetilde{c}$, such that for all $c<\widetilde{c}$ this is true. Define, for $\forall$ $g \in \Gamma, \forall i \in N, \forall j \in N: \delta=\min _{g, i, j}\left\{\pi_{i}(g+i j)-\pi_{i}(g) \mid \pi_{i}(g+i j)>\pi_{i}(g)\right\}$
}

Since the maximum amount of links a firm can form is $N-1$, then let $\widetilde{c}=\frac{\delta}{N-1}$. 
to analyze whether, given a network $g \neq g^{c}$, there is always an incentive to continue forming or deleting links. We show that, to the contrary, there can always exist a set of firms who remain outsiders for any additional link they are offered.

Theorem 1 When $\phi$ is large enough, there exists asymmetric pairwise stable REDD networks which have the dominant group structure

Proof: See Appendix B.

As a consequence, even when costs of linking are negligible the complete network will not always be formed. Interestingly, this occurs when cooperation is of high strategic importance (high $\phi$ ).

Theorem 1 states that the other possible pairwise stable networks are the dominant group structure where firms outside the main component do not participate to the contest. We now provide a condition which characterizes all pairwise stable dominant group networks.

Proposition 2 Let $g^{k}$ be a dominant group network where the main component is formed by $k$ firms. Then, $g^{k}$ is pairwise stable if and only if the following condition holds

$$
\phi\left[(N-k-1)+(k-1) \frac{1}{\phi^{k-1}}+\frac{1}{\phi^{k}}\right]<N-2
$$

Proof: See Appendix C.

Naturally, if more than one equilibrium exists, we need to analyze if we can rank the various equilibria using the pareto dominance criterion. Unfortunately, the next proposition shows that the complete network can pareto dominate a dominant group network and vice versa:

Proposition 3 Let $g^{\underline{k}}, \ldots g^{k} \ldots g^{N-1}$ be all pairwise stable dominant group networks. Then, the complete network $g^{c}$ pareto dominates such networks if and only if

$$
\phi>\max _{k}\left(\sqrt[N-k]{\frac{(N+1) N}{(k+1) k}}\right) \quad \forall k=\underline{k} \ldots N-1
$$

Proof: See Appendix D.

In the next section we propose an example that illustrates our model. 


\section{An example}

Suppose there are $\mathrm{N}=5$ firms that want to discover a new technology. Some possible network structures that can be formed are shown in Figure 1. The first step to solve the model is to obtain the maximal ex-ante benefits $\alpha_{i}$ that firms can get in each network (See Figure 2 for an example). Then, since less connected firms have a lower payoff (Corollary 1) and consequently a higher chance of not participating to the contest, we check when they satisfy their participation constraint. For example, looking at network $g^{3}$ (Figure 2) we first calculate the participation constraints of firms 4 and 5. If they are satisfied, we then calculate the payoffs of the remaining firms knowing that all firms will participate to the race (i.e. $f\left(g^{3}\right)=5$ ). If firms 4 and 5 do not participate, we calculate the payoff of the other firms knowing that $f\left(g^{3}\right)=3$

\subsection{Pairwise Stability (PWS)}

From the previous section we know that the possible pairwise stable networks are the complete network $g^{c}$ and the dominant group networks $g^{k}$ where $k=2,3,4$ (Figure 3 ). Theorem 1 ensures that the complete network is always PWS while Proposition 2 allows us to verify when asymmetric dominant group networks are PWS. In particular

1. $g^{2}$ is PWS if and only if

$$
\phi\left[2+\frac{1}{\phi}+\frac{1}{\phi^{2}}\right]<3 \Leftrightarrow 2 \phi+\frac{1}{\phi}<2
$$

2. $g^{3}$ is PWS if and only if

$$
\phi\left[1+\frac{2}{\phi^{2}}+\frac{1}{\phi^{3}}\right]<3 \Leftrightarrow \phi+\frac{2}{\phi}+\frac{1}{\phi^{2}}<3 \Leftrightarrow \phi^{3}-3 \phi^{2}+2 \phi+1<0
$$

3. $g^{4}$ is PWS if and only if

$$
\phi\left[\frac{3}{\phi^{3}}+\frac{1}{\phi^{4}}\right]<3 \Leftrightarrow 3 \phi^{3}-3 \phi-1>0
$$

It is straightforward to see that PWS conditions for $g^{2}$ and $g^{3}$ are never satisfied while $g^{4}$ is PWS when $\phi$ is large enough $\left(\phi>\underline{\phi_{4}} \simeq 1.137158\right)$. Therefore in our example there are two PWS networks

- $g^{c}$ for any $\phi>1$

- $g^{4}$ for any $\phi \geq \underline{\phi_{4}}$

Moreover, following Proposition 3, the complete network pareto dominates $g^{4}$ only when $\phi>3 / 2$. 


\section{$6 \quad$ Strong Stability (SS)}

The example shows us that, for a considerable range of parameters, multiple equilibria coexist. One can wonder whether it is possible to eliminate some of the equilibria by looking at refinements of the pairwise stability concept. We do so by studying strong stability which demands pairwise stable networks to be immune to coalitional deviations. When one pairwise stable network pareto dominates another one, it is clear that there exists a coalitional deviation from one to the other. ${ }^{11}$ Unfortunately, as we will see below, our above example conveys that there need not exist any strongly stable network.

Lemma 1 There need not to be any strongly stable network

\subsection{An example (continued)}

Recall that, a network $g$ is SS when there is no coalition of players that finds it profitable to deviate from $g$ to another network $g^{\prime}$ (no matter whether $g^{\prime}$ is pairwise stable or not). Let us use the following notation

$$
g^{X} \stackrel{\{i, j, k\}}{\longrightarrow} g^{Y}
$$

to indicate that players $i, j$ and $k$ deviate from network $g^{X}$ toward $g^{Y}$.

Strong Stability of network $g^{c}$ If the deviation $g^{c} \stackrel{\{1,2,3,4\}}{\longrightarrow} g^{4}$ is profitable, then the complete network is not SS. In order to verify such profitability we must distinguish two cases.

- If the deviation does not lead firm 5 to drop out of the race (i.e. when $1<\phi<\sqrt[3]{4 / 3})$ ), then deviating players $i=1,2,3,4$ are better off if and only if

$$
\Pi_{i}\left(g^{4}\right)-\Pi_{i}\left(g^{c}\right)>0 \Leftrightarrow \frac{\phi^{9}}{2\left(\phi^{3}+2\right)\left(\phi^{3}+4\right)}-\frac{\phi^{4}}{30}>0
$$

Plotting such difference within the range $1<\phi<\sqrt[3]{4 / 3}$ ) (Figure 5) we can immediately see that it is always satisifed. Consequently $g^{c}$ is not SS in such range because it is always profitable to deviate from $g^{c}$ to $g^{4}$.

- On the other hand if the deviation forces firm 5 to leave the race (i.e. $\phi \geq \sqrt[3]{4 / 3})$ then $g^{c} \stackrel{\{1,2,3,4\}}{\longrightarrow} g^{4}$ is profitable if and only if

$$
\Pi_{i}\left(g^{4}\right)>\Pi_{i}\left(g^{c}\right) \Leftrightarrow \frac{\phi^{3}}{20}>\frac{\phi^{4}}{30} \Leftrightarrow \phi<\frac{3}{2}
$$

\footnotetext{
${ }^{11}$ Looking at our example this means that $g^{4}$ is never $\mathrm{SS}$ as $\phi>3 / 2$ because a grand coalition can profitably deviate to the complete network.
} 
Therefore, whenever $\sqrt[3]{4 / 3} \leq \phi<\frac{3}{2}$ the deviation $g^{c \stackrel{\{1,2,3,4\}}{\longrightarrow}} g^{4}$ is profitable too.

Merging the two cases we have that the complete network is not SS for any $1 \leq \phi<\frac{3}{2}$. Moreover, one can prove that, whenever $3 / 2<\phi<1.89414$, three firms will always find it profitable to deviate from the full network to a network $g^{\widetilde{k}}$ with two components of three and two firms respectively. ${ }^{12}$ Therefore, even though the complete network pareto dominates $g^{4}$ it is still not SS when $3 / 2<$ $\phi<\widehat{\phi}=1.89414$.

Strong Stability of network $g^{4} \quad$ About the strong stability of $g^{4}$, note that since $g^{4}$ is not PWS while $g^{c}$ is not SS as $1<\phi<\phi_{4}$, this means that there is no SS network in this range.

However we know that $g^{4}$ is PWS for any $\phi \geq \phi_{4}$. Is it possible that $g^{4}$ is SS when $\underline{\phi_{4}} \leq \phi<\frac{3}{2}$ ? To answer this question let consider the following deviation $g^{4} \stackrel{\{1,2,3\}}{\longrightarrow} g^{3}$.

- If $\underline{\phi_{4}} \leq \phi<\sqrt{\frac{3}{2}}$, outsiders of the component participate. Therefore, the deviation $g^{4} \stackrel{\{1,2,3\}}{\longrightarrow} g^{3}$ is profitable if and only if

$$
\Pi_{i}\left(g^{3}\right)-\Pi_{i}\left(g^{4}\right) \geq 0 \Leftrightarrow \frac{\phi^{2}\left(2 \phi^{2}-1\right)^{2}}{\left(4 \phi^{2}+2\right)\left(2 \phi^{2}+3\right)}-\frac{\phi^{3}}{20}>0
$$

Plotting such difference for any value of $\phi$ inside $\left[\phi_{4}, \sqrt{3 / 2}\right]$ we can immediately see that it is always satisfied (Figure 4). Therefore, as $\phi \in\left[\phi_{4}, \sqrt{\frac{3}{2}}\right]$ network $g^{4}$ is PWS but not strongly stable.

- On the other hand, outsiders do not participate as $\sqrt{\frac{3}{2}} \leq \phi<\frac{3}{2}$. Therefore, a deviation to $g^{3}$ is profitable only when

$$
\Pi_{i}\left(g^{3}\right)>\Pi_{i}\left(g^{4}\right) \Leftrightarrow \frac{\phi^{2}}{12}>\frac{\phi^{3}}{20} \Leftrightarrow \phi<\frac{5}{3}
$$

which means that it is always profitable to deviate from $g^{4}$ to $g^{3}$ whenever $\sqrt{\frac{3}{2}} \leq \phi<\frac{3}{2}$.

As a consequence of these results network $g^{4}$ is PWS but never SS when $\underline{\phi_{4}} \leq \phi<\frac{3}{2}$.

\footnotetext{
${ }^{12}$ This is due to the fact that in such network both outsiders always participate so that the other three firms will deviate only when

$$
\Pi_{i}\left(g^{\widetilde{k}}\right)>\Pi_{i}\left(g^{c}\right) \Leftrightarrow \frac{\phi^{2}(2 \phi-1)^{2}}{(4 \phi+2)(2 \phi+3)}>\frac{\phi^{3}}{20}
$$

a condition that is satisfied as $3 / 2<\phi<1.89414$.
} 
Given that $g^{4}$ and $g^{c}$ are never SS while all the other networks are not PWS, there is no strongly stable network whenever $\phi \in[1, \widehat{\phi}]$. Such result clearly indicates that strong stability may be too strong a refinement with respect to pairwise stability.

\section{Concluding Remarks}

In this paper we provide a theoretical background to sectors featured by R\&D "co-opetition", namely sectors where firms cooperate in R\&D even though they compete in a patent contest. Our analysis started from the observation that, during the last years, there has been an increasing number of weak partnerships among firms that compete for the development of new products/technologies.

In this setting we studied, by using an all-pay auction approach, how this weak collaboration affects strategic decisions during the patent contest itself, and how the latter influences the possible network structures the firms can hope to form. The all pay auction approach allows us to 1) endogenize both network formation and R\&D intensities and 2) take heterogeneous and private valuations for patents into account.

We find that, different from previous literature, the complete network is not always the only pairwise stable network, even and especially if the benefits from cooperating are important. Interestingly, the other possible stable networks all have the realistic property that some firms decide not to participate in the contest. In other words, network formation can serve as an endogenous barrier to entry.

We further show that there need not be any network that survives a well known refinement of pairwise stability: strong stability, which imposes networks to be immune to coalitional deviations.

\section{A Proof of Lemma 1}

Let us consider the following firm $i$ 's profits in the network $g$

$$
\Pi_{i}(g)=\frac{\alpha_{i}\left(\alpha_{i}\left(A_{p}+\frac{1}{\alpha_{j}}\right)-f(g)+2\right)^{2}}{\left(2 \alpha_{i}\left(A_{p}+\frac{1}{\alpha_{j}}\right)-f(g)+3\right)\left(1+\alpha_{i}\left(A_{p}+\frac{1}{\alpha_{j}}\right)\right)}
$$

and in the network $g+i j$

$$
\Pi_{i}(g+i j)=\frac{\phi \alpha_{i}\left(\phi \alpha_{i}\left(A_{p}+\frac{1}{\phi \alpha_{j}}\right)-f(g+i j)+2\right)^{2}}{\left(2 \phi \alpha_{i}\left(A_{p}+\frac{1}{\phi \alpha_{j}}\right)-f(g+i j)+3\right)\left(1+\phi \alpha_{i}\left(A_{p}+\frac{1}{\phi \alpha_{j}}\right)\right)}
$$


where $\phi>1$. Suppose for simplicity that $f(g)=f(g+i j)=f \leq N$, namely that no firm decides not to participate. ${ }^{13}$ Taking the difference of the log-profits, a new link is profitable when

$$
\begin{aligned}
& \log \Pi_{i}(g+i j)-\log \Pi_{i}(g)=\log \frac{\phi \alpha_{i}}{\alpha_{i}}+2 \log \frac{\phi \alpha_{i}\left(A_{p}+\frac{1}{\phi \alpha_{j}}\right)-f+2}{\alpha_{i}\left(A_{p}+\frac{1}{\alpha_{j}}\right)-f+2} \\
& -\log \frac{2 \phi \alpha_{i}\left(A_{p}+\frac{1}{\phi \alpha_{j}}\right)-f+3}{2 \alpha_{i}\left(A_{p}+\frac{1}{\alpha_{j}}\right)-f+3}-\log \frac{1+\phi \alpha_{i}\left(A_{p}+\frac{1}{\phi \alpha_{j}}\right)}{1+\alpha_{i}\left(A_{p}+\frac{1}{\alpha_{j}}\right)}
\end{aligned}
$$

Simplifying some terms we have

$$
\begin{aligned}
& \log \Pi_{i}(g+i j)-\log \Pi_{i}(g)=\log \phi+2 \log \frac{\phi \alpha_{i} A_{p}+\frac{\alpha_{i}}{\alpha_{j}}-f+2}{\alpha_{i} A_{p}+\frac{\alpha_{i}}{\alpha_{j}}-f+2} \\
& -\log \frac{2 \phi \alpha_{i} A_{p}+\frac{\alpha_{i}}{\alpha_{j}}-f+3}{2 \alpha_{i} A_{p}+\frac{\alpha_{i}}{\alpha_{j}}-f+3}-\log \frac{1+\phi \alpha_{i} A_{p}+\frac{\alpha_{i}}{\alpha_{j}}}{1+\alpha_{i} A_{p}+\frac{\alpha_{i}}{\alpha_{j}}}
\end{aligned}
$$

Rewriting $\phi=1+\psi$ and simplifying

$$
\begin{aligned}
& \log \Pi_{i}(g+i j)-\log \Pi_{i}(g)=\log (1+\psi)+2 \log \left[1+\frac{\psi \alpha_{i} A_{p}}{\alpha_{i} A_{p}+\frac{\alpha_{i}}{\alpha_{j}}-f+2}\right] \\
& -\log \left[1+\frac{2 \psi \alpha_{i} A_{p}}{2 \alpha_{i} A_{p}+\frac{\alpha_{i}}{\alpha_{j}}-f+3}\right]-\log \left[1+\frac{\psi \alpha_{i} A_{p}}{1+\alpha_{i} A_{p}+\frac{\alpha_{i}}{\alpha_{j}}}\right]
\end{aligned}
$$

Rearranging terms

$$
\begin{gathered}
\log \Pi_{i}(g+i j)-\log \Pi_{i}(g)=\overbrace{\log \left[1+\frac{\psi \alpha_{i} A_{p}}{\alpha_{i} A_{p}+\frac{\alpha_{i}}{\alpha_{j}}-f+2}\right]-\log \left[1+\frac{\psi \alpha_{i} A_{p}}{1+\alpha_{i} A_{p}+\frac{\alpha_{i}}{\alpha_{j}}}\right]}^{A}+ \\
\underbrace{\log (1+\psi)+\log \left[1+\frac{\psi \alpha_{i} A_{p}}{\alpha_{i} A_{p}+\frac{\alpha_{i}}{\alpha_{j}}-f+2}\right]-\log \left[1+\frac{2 \psi \alpha_{i} A_{p}}{2 \alpha_{i} A_{p}+\frac{\alpha_{i}}{\alpha_{j}}-f+3}\right]}_{B}
\end{gathered}
$$

it is sufficient to prove that A and B are always positive.

A First, $\mathrm{A}$ is positive if and only if

$$
\begin{gathered}
\log \left[1+\frac{\psi \alpha_{i} A_{p}}{\alpha_{i} A_{p}+\frac{\alpha_{i}}{\alpha_{j}}-f+2}\right] \geq \log \left[1+\frac{\psi \alpha_{i} A_{p}}{1+\alpha_{i} A_{p}+\frac{\alpha_{i}}{\alpha_{j}}}\right] \Rightarrow \\
\frac{\psi \alpha_{i} A_{p}}{\alpha_{i} A_{p}+\frac{\alpha_{i}}{\alpha_{j}}-f+2} \geq \frac{\psi \alpha_{i} A_{p}}{1+\alpha_{i} A_{p}+\frac{\alpha_{i}}{\alpha_{j}}} \Rightarrow 1+\alpha_{i} A_{p}+\frac{\alpha_{i}}{\alpha_{j}} \geq \alpha_{i} A_{p}+\frac{\alpha_{i}}{\alpha_{j}}-f+2 \Rightarrow f-1 \geq 0
\end{gathered}
$$

\footnotetext{
${ }^{13}$ Since such assumption excludes the indirect benefit that some competitors could give up because of this new link, we are actually considering the least favorable condition for the profitability of the new link $i j$.
} 
B Therefore A is always positive. Now, in order to have B positive we need that

$$
\log (1+\psi)+\log \left[1+\frac{\psi \alpha_{i} A_{p}}{\alpha_{i} A_{p}+\frac{\alpha_{i}}{\alpha_{j}}-f+2}\right] \geq \log \left[1+\frac{2 \psi \alpha_{i} A_{p}}{2 \alpha_{i} A_{p}+\frac{\alpha_{i}}{\alpha_{j}}-f+3}\right]
$$

A simple way to check this condition is to see when the right hand side is dominated by either the first term or the second one of the left hand side. The LHS first term dominates the RHS when ${ }^{14}$

$$
\begin{gathered}
\log (1+\psi) \geq \log \left[1+\frac{2 \psi \alpha_{i} A_{p}}{2 \alpha_{i} A_{p}+\frac{\alpha_{i}}{\alpha_{j}}-f+3}\right] \Leftrightarrow \psi \geq \frac{2 \psi \alpha_{i} A_{p}}{2 \alpha_{i} A_{p}+\frac{\alpha_{i}}{\alpha_{j}}-f+3} \Leftrightarrow \\
2 \alpha_{i} A_{p}+\frac{\alpha_{i}}{\alpha_{j}}-f+3 \geq 2 \alpha_{i} A_{p} \Leftrightarrow \frac{\alpha_{i}}{\alpha_{j}} \geq f-3
\end{gathered}
$$

On the other hand the second term of the LHS dominates the RHS when

$$
\begin{aligned}
& \log \left[1+\frac{\psi \alpha_{i} A_{p}}{\alpha_{i} A_{p}+\frac{\alpha_{i}}{\alpha_{j}}-f+2}\right] \geq \log \left[1+\frac{2 \psi \alpha_{i} A_{p}}{2 \alpha_{i} A_{p}+\frac{\alpha_{i}}{\alpha_{j}}-f+3}\right] \Leftrightarrow \\
& \frac{\psi \alpha_{i} A_{p}}{\alpha_{i} A_{p}+\frac{\alpha_{i}}{\alpha_{j}}-f+2} \geq \frac{2 \psi \alpha_{i} A_{p}}{2 \alpha_{i} A_{p}+\frac{\alpha_{i}}{\alpha_{j}}-f+3} \Leftrightarrow \\
& 2 \alpha_{i} A_{p}+\frac{\alpha_{i}}{\alpha_{j}}-f+3 \geq 2\left(\alpha_{i} A_{p}+\frac{\alpha_{i}}{\alpha_{j}}-f+2\right) \Leftrightarrow \frac{\alpha_{i}}{\alpha_{j}} \leq f-1
\end{aligned}
$$

This means that, for any value of $f$, the first and/or the second term of the left hand side dominate(s) the RHS. Therefore B is always positive. Concluding, since A and B are always positive, it is always beneficial to form a link with another partner.

\section{B Proof of Theorem 1}

First, by Lemma 1 we know that all participating firms will always form a complete component. Second, a firm $i$ will not form a link when

$$
\Pi_{i}(N) \geq 0 \Leftrightarrow(N-2) \geq \alpha_{i} \sum_{j \neq i} \alpha_{j}^{-1}
$$

It is straightforward to see that when two competitors form a new link they reduce the right hand side of the participation constraint while the left hand side is constant. Therefore, as $\phi$ is high enough and firms $j \neq i$ are sufficiently linked, it is possible that firm $i$ does not participate in the network $g+i j$ for any $j$ participating firm. Finally, equation 1 implies that a non participating firm $i$ always has an incentive to stay isolated. Q.E.D.

\footnotetext{
${ }^{14}$ Note that since the participation condition for firm $i$ requires that $\alpha_{i} A_{p}+\frac{\alpha_{i}}{\alpha_{j}} \geq N-2$, the second term of the LHS is always positive.
} 


\section{Proof of Proposition 2}

Let us consider a network $g^{k}$ where the dominant group consists of $k$ firms while all the others are isolated and non participating firms. In order not to participate outsider firms must not only violate their participation constraint

$$
\left[(N-k-1)+\frac{k}{\phi^{k-1}}\right]<N-2
$$

but they also have to stay out even when they can form a new link

- with a member of the component

$$
\phi\left[(N-k-1)+(k-1) \frac{1}{\phi^{k-1}}+\frac{1}{\phi^{k}}\right]<N-2
$$

- with another outsider

$$
\phi\left[(N-k-2)+k \frac{1}{\phi^{k-1}}+\frac{1}{\phi}\right]<N-2
$$

Network $g^{k}$ is PWS only when all these conditions hold. However, we can show that Equation (16) is the most restrictive.

First, Equation(16) is more restrictive than the participation constraint if and only if

$$
\begin{gathered}
\phi\left[(N-k-1)+(k-1) \frac{1}{\phi^{k-1}}+\frac{1}{\phi^{k}}\right]>\left[(N-k-1)+\frac{k}{\phi^{k-1}}\right] \Leftrightarrow \\
(\phi-1)(N-k-1)+\frac{k-1}{\phi^{k-2}}>\frac{k-1}{\phi^{k-1}} \Leftrightarrow(\phi-1)(N-k-1) \phi^{k-1}+(k-1)(\phi-1)>0 \Leftrightarrow \\
(\phi-1)\left[(N-k-1) \phi^{k-1}+(k-1)\right]>0
\end{gathered}
$$

a condition that is always satisfied.

On the other hand, Equation(16) is more restrictive than Equation (17) if and only if

$$
\begin{aligned}
& \phi\left[(N-k-1)+(k-1) \frac{1}{\phi^{k-1}}+\frac{1}{\phi^{k}}\right]>\phi\left[(N-k-2)+k \frac{1}{\phi^{k-1}}+\frac{1}{\phi}\right] \Leftrightarrow \\
& 1+\frac{1}{\phi^{k}}>\frac{1}{\phi^{k-1}}+\frac{1}{\phi} \Leftrightarrow \phi^{k}+1>\phi+\phi^{k-1} \Leftrightarrow \phi^{k-1}(\phi-1)>(\phi-1) \Leftrightarrow \\
& \left(\phi^{k-1}-1\right)(\phi-1)>0
\end{aligned}
$$

which is always true. Therefore, Equation(16) is the most restrictive condition for the PWS of a dominant group network.Q.E.D. 


\section{Proof of Proposition 3}

It is straightforward to see that moving from a dominant group network to the complete one, firms that were outside the component are better off insofar as their payoff becomes strictly positive instead of being null. Therefore, the complete network pareto dominates a dominant group network $g^{k}$ only when all component members $i$ are better off when they move from $g^{k}$ to $g^{c}$, namely

$$
\Pi_{i}\left(g^{c}\right)>\Pi_{i}\left(g^{k}\right) \Leftrightarrow \frac{\phi^{N-1}}{(N+1) N}>\frac{\phi^{k-1}}{(k+1) k} \Leftrightarrow \phi>\sqrt[N-k]{\frac{(N+1) N}{(k+1) k}}
$$

Consequently, the complete network pareto dominates all the other PWS networks only when Equation (18) holds for any $k=\underline{k}, \ldots, N-1$. Q.E.D. 


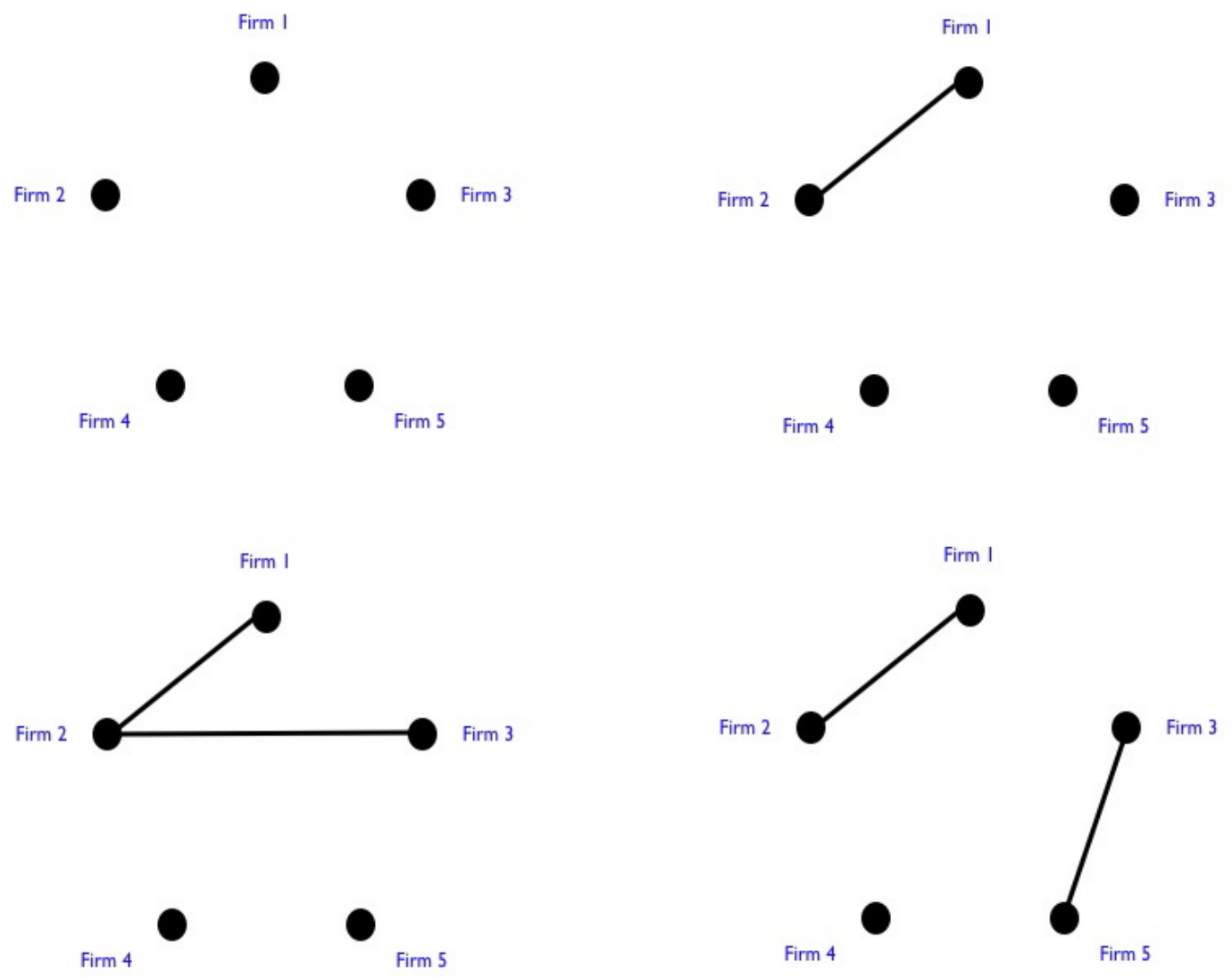

Figure 1: Examples of networks with 5 firms

\section{E Figures}



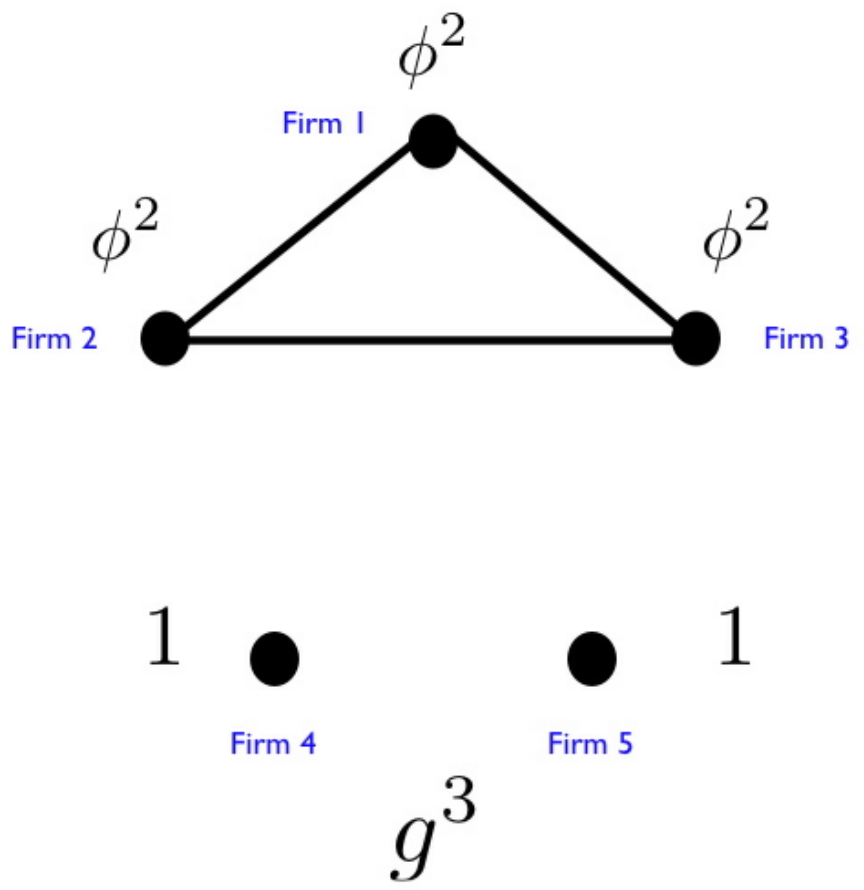

Figure 2: Firms' maximal ex ante benefits $\alpha_{i}$ in network $g^{3}$ 

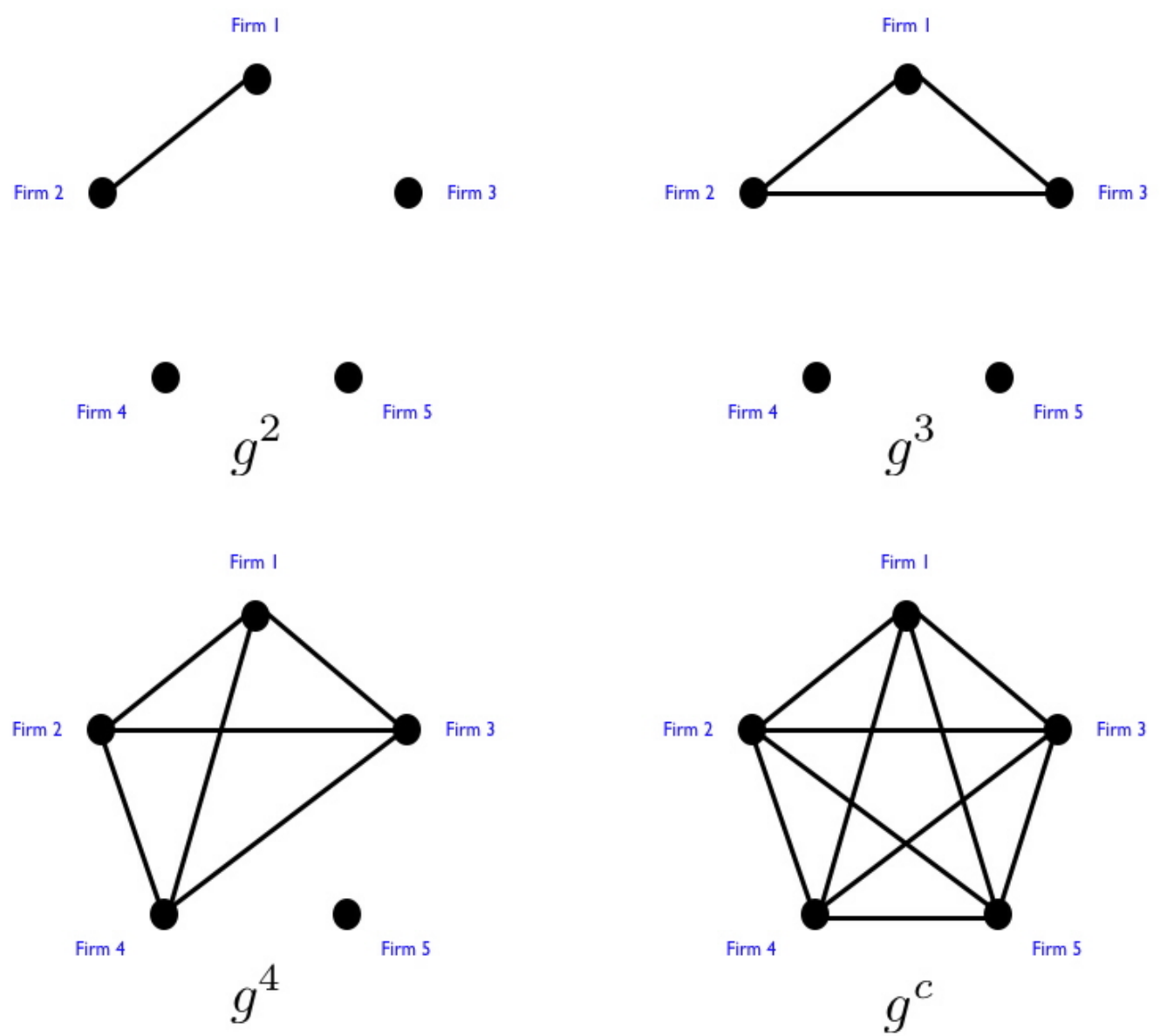

Figure 3: Dominant group networks and the complete network. 


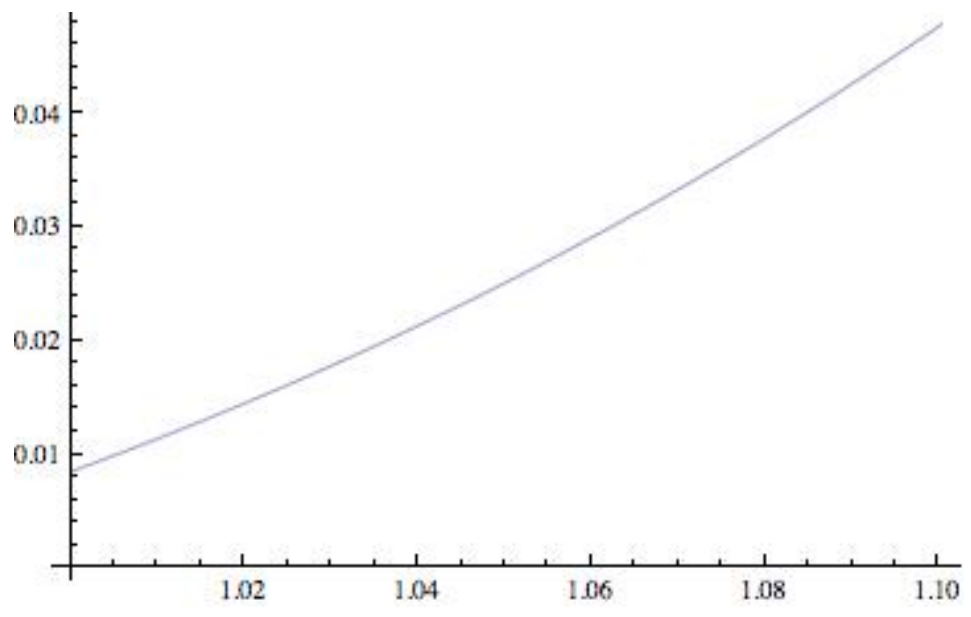

Figure 4: $\Pi_{i}\left(g^{4}\right)-\Pi_{i}\left(g^{c}\right)$ as $\phi \in[1, \sqrt[3]{4 / 3}]$

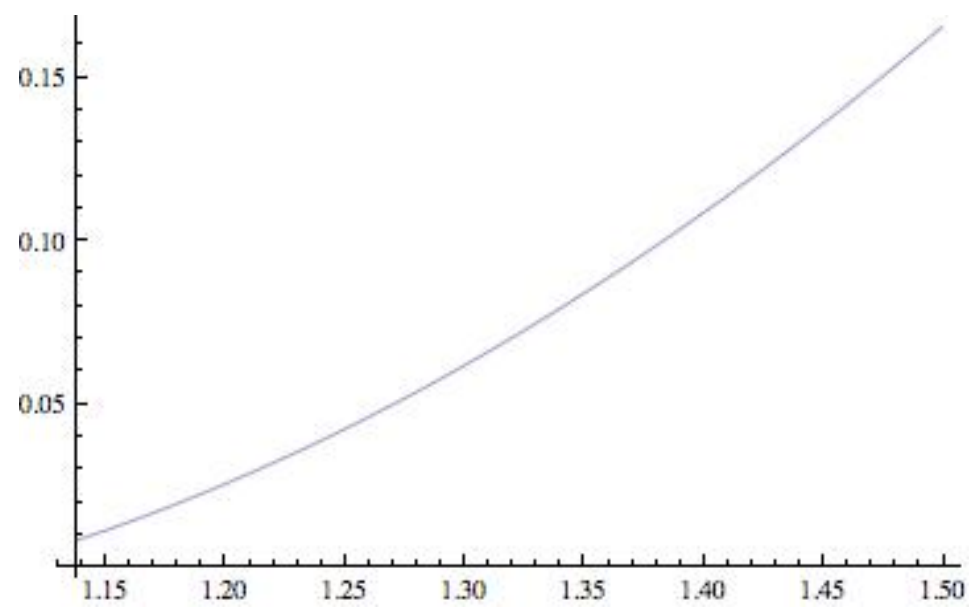

Figure $5: \Pi_{i}\left(g^{3}\right)-\Pi_{i}\left(g^{4}\right)$ as $\phi \in\left[\phi_{4}, 3 / 2\right]$ 


\section{References}

[2000] Anand B. N., and Khanna T., "The Structure of Licensing Contracts", The Journal of Industrial Economics, 48 (1), 103-135.

[2003] Baye M.R. and Hoppe H. C., "The strategic equivalence of rent-seeking, innovation, and patent race games", Games and Economic Behavior, 44 (2), 217-226.

[2003] Caloghirou Y., Ioannides S. and Vonortas N.S., "Research Joint Ventures", Journal of Economic Surveys, 17 (4), 541-570.

[2002] Cohen W., Goto A., Nagata A., Nelson R., and Walsh J., "R\&D Spillovers, Patents and the Incentives to Innovate in Japan and United States", Research Policy, 31 (8-9), 1349-1367.

[2006] Goyal S. and Joshi S., "Unequal Connections", International Journal of Game Theory, 34 (3), 319-349.

[2005] Greenlee P., "Endogenous Formation of Competitive Research Sharing Joint Venture", The Journal of Industrial Economics, 53 (3), 355-391.

[1999] Hagerdoon J., Link A. N. and Vonortas N. S., "Research Partnerships", Research Policy, 29 (4-5), 567-586.

[1989] A L Hillman, A.L. and Riley J.G., "Politically contestable rents and transfers", Economics and Politics, 1, 17-39.

[2005] Jackson M. and van den Nouweland A., "Strongly Stable Networks", Games and Economic Behavior, 51 (2), 420-444.

[1996] Jackson M. and Wolinsky A., "A Strategic Model of Social and Economic Networks", Journal of Economic Theory, 71 (1), 44-74.

[2008] Joshi S., "Endogenous formation of coalitions in a model of a race", Journal of Economic Behavior \&3 Organization, 65 (1), 62-85.

[1979] Loury G.C., "Market Structure and Innovation", Quarterly Journal of Economics, 71 (1), 44-74.

[1995] Martin S. R\&D joint ventures and tacit product market collusion European Journal of Political Economy, 11 (4), 733-741.

[2002] Martin S. Spillovers, Appropriability, and R\&D Journal of Economics, 75 (1), 1-32. 
[2001] OECD, Directorate for Financial, Fiscal and Enterprise Affairs Comitee on Competition Law and Policy, Competition Issues in Joint Ventures.

[2006] Parreiras S. O. and Rubinchik A. "Contests with Heterogeneous Agents", CORE Discussion Papers, No.4, Université Catholique de Louvain, Belgium.

[2006] Roijakkers N., and Hagerdoon J., "Inter-firm R\&D partnering in pharmaceutical biotechnology since 1975: Trends, patterns, and networks", Research Policy, 35 (1), 431-446.

[2008] Stein J. C., "Conversations among Competitors", The American Economic Review, 98 (5), 2150-2162.

[2006] United Nations Industrial Development Organization, Alliances and joint ventures: Patterns of internationalization for developing country enterprises. Part one, 2006. 